\title{
The Pattern of Dermatological Disorders among Patients Attending the Skin O.P.D of A Tertiary Care Hospital in Kolkata, India.
}

\author{
Dr Baijayanti Baur ${ }^{1}$, Dr Jhuma Sarkar ${ }^{1,}$ Dr Nirmalya Manna ${ }^{1,}$ \\ Dr Lina Bandyopadhyay ${ }^{2}$ \\ ${ }^{1}$ Department of Community Medicine, Medical College, Kolkata, India \\ ${ }^{2}$ All India Institute of Hygiene \& Public Health
}

\begin{abstract}
Pattern of skin diseases vary from region to region. Types of skin diseases are influenced by various factors and the consequent morbidities have a profound effect on individual and the community. An observational cross-sectional hospital based study was carried out in the dermatology outpatient department of Calcutta National Medical College, India to find out the pattern of skin diseases and its socio-demographic and environmental correlates. Maximum number of study population was aged 15-24 years (35.6\%) and female gender (51.5\%). Scabies was most common infectious type (20.4\%) and acne (12.4\%) was most common noninfectious dermatoses among the study population. Tinea and Acne most commonly recurred among the study population. Maintenance of personal hygiene $(71.3 \%)$ and sufficient availability of water at household level (91.8\%) were more among non-infectious group of patients in comparison to infectious group. Socio-economic status, tobacco use, over-crowding and positive history of contact were significantly associated with infectious type of dermatoses. Health education especially interpersonal communication can alleviate some of the miseries of dermatoses in individual and community.
\end{abstract}

Keywords: Dermatoses, infectious group, non-infectious group, environmental factors

\section{Introduction}

Skin diseases affect all ages from neonate to the elderly. It causes harm in a number of ways and can have a profound effect on both the individual and the community. Morbidity is significant through disfigurement, disability or symptoms such as intractable itch, as is the reduction in quality of life, even social isolation and economic burden. Death, though rare but still seen from metastatic skin cancer. Many times, some dermatological manifestations may give some clue to the presence of benign or malignant systemic diseases in individual. Despite the high frequency of certain skin diseases in developing countries, they have so far not been regarded as a significant health problem in the development of public health strategy. Indeed some less common health problems in the same countries receive more attention. ${ }^{1,2,3,4}$

Pattern of skin diseases vary from country to country. Even in the same country it differs from region to region. Types of skin diseases are influenced by various factors like genetic, race, religion, occupation, nutrition, habits etc. Geographical factors such as season and climate also contribute to the increased prevalence of certain type of skin disorder in a particular area. India is such a country where wide variation in climate, socio-economic status, religion, and customs is quite prevalent in different parts of the country. In developing countries, other than hot and humid climatic condition, low hygiene, poor access to water, overcrowding, high interpersonal contact also play significant etiological role for certain skin diseases like pyoderma, scabies,fungal infection. ${ }^{3,5,6,7,8}$

Considering all these factors this study was carried out with the following objectives;

1) To find out the pattern of skin diseases among the patients attending the skin O.P.D of a tertiary care hospital in Kolkata.

2) To assess the relation of demographic, socioeconomic and environmental factors with the type of dermatological disorders.

\section{Methodology}

A hospital based cross-sectional observational study was carried out in the Skin O.P.D of Calcutta National Medical College \& Hospital in West Bengal, India. Data were collected from $14^{\text {th }}$ May-12 $2^{\text {th }}$ June, 2010. Patients who were attending the Skin O.P.D during data collection period and were willing to participate constituted the study population. In case of patients aged $\leq 14$ years accompanying guardians were interviewed. Before data collection Institutional Ethics Committee clearance and permission from Hospital Superintendant 
were taken. Informed verbal consent was taken from the interviewed patients. This study was in accordance with Helsinki declaration.

Data were collected in the predesigned, pretested, semi-structured schedule by exit interview. Dermatological diagnosis by qualified dermatologists from the O.P.D tickets was recorded. Patients had doubtful diagnosis was excluded from the study population. The schedule included socio-demographic factors like age, sex, residence, religion, socio-economic factors and personal characteristics like habits, hygiene. Socioeconomic scale was determined by using B.G Prasad scale. ${ }^{9}$ Current user of tobacco was defined as someone who at the time of survey smokedlused tobacco in any form either daily or occasionally. The group of non-users comprised individuals who were never used (those who have never smokedlchewed tobacco at all) and exsmokerslchewer. Time taken for completing the schedule was on average 15 minutes. Daily O.P.D. attendance in this clinic was 50-60 per day. During O.P.D. hours 8-10 patients per day were interviewed. By systematic random sampling every fifth patients were interviewed after taking consent. Total 231 patients were interviewed. Six patients had doubtful diagnosis (needed laboratory investigations), therefore excluded from final analyses. Finally 225 patients were analyzed using EpiInfo version 3.5.1 by descriptive statistics and $\chi^{2}$ when needed.

\section{Results}

The socio-demographic characteristics of the interviewed patients are depicted in Table 1. It showed child population i.e patients aged $\leq 14$ years constituted $19.5 \%$ of the study population. Maximum no. of study population were aged $15-24$ yrs(35.6\%),followed by $25-34$ yrs.(22.2\%). Female patients were more $(51.5 \%)$ than their male counterpart. Muslims constituted $55.2 \%$ of the study population followed by Hindus $44.4 \%$. Patients having secondary education $(47.6 \%)$ were more in this study population. Illiterate were $20.0 \%$ and $15.5 \%$ patients had primary school education. One-fourth $(25.8 \%)$ of the study population were housewives, closely followed by students (23.5\%).Unskilled worker were 14.7\%.Predominant Socio-economic category was class III \& IV(35.1\% each) followed by class V(13.4\%). Table 2 showed pattern of skin diseases among the interviewed patients. Non Infectious dermatoses were more than Infectious group(54.3\% vs. $45.7 \%)$.Among the Infectious category, Scabies was most common type (20.4\%).Next common group were Tinea infection (10.7\%), followed by Impetigo (3.6\%), Folliculitis (2.2\%), Pyoderma (1.8\%),Boils (1.3\%).Leprosy patients were $2.7 \%$ of the studied patients. For Non-Infectious dermatoses Acne (12.4\%) was more common followed by Dermatitis (9.8\%), Eczema (7.1\%), Insect bite (3.1\%),Urticaria \& Psoriases $(2.7 \%)$ each. It was evident from Table 3 that among the Infectious group Folliculitis(40.0\%) followed by Tinea infection (37.5\%) were the common causes of recurrent visits in this Dermatology O.P.D. Scabies $(17.4 \%)$ and Impetigo(12.5\%) were the other common reasons for recurrent visits. For Non-Infectious group 37.5\% Eczema patients visited recurrently. Miliaria Rubra (33.3\%), Acne (32.2\%), Urticaria (16.7\%) were other causes of recurrent visits. Table 4 showed that among studied patients, $14.3 \%$ patients came from Rural area. Proportion of rural patients were more in infectious group than non infectious group $(16.5 \%$ vs.12.3\%).In the infectious dermatoses more patients came from urban area $(44.7 \%)$ followed by urban slum $(38.8 \%)$.For non-infectious category more patients came from urban slum (46.7\%) followed by urban (41.0\%). But this relation of type of skin diseases and residential area was not significant. In the socio-economic class I \&II and also IV \&V proportion of infectious patients were more. But in Socio-Economic class III non infectious patients were more (Non Infectious-41.0\% vs.Infectious$28.1 \%)$.This relation of type of skin disease with socio-economic class was also statistically significant(p.03). Among the studied patients, $23.5 \%$ were current user of Tobacco. Infectious dermatoses were more common among tobacco user $(32.0 \%$ vs. $16.4 \%)$, which was also significant statistically $(\mathrm{p}=0.00)$. Contact history was given by $20.9 \%$ of the patients, where significantly $(\mathrm{p}=0.000)$ more proportion of Infectious group had this history. Majority of the studied group (92.9\%) availed sufficient water. Infectious diseases were more common among those who had not got sufficient water (infectious-9.7\%, non infectious-8.2\%) but this was not significant. Personal hygiene was maintained by $66.2 \%$ of the patients. Infectious dermatoses were more (39.8\%) among those who had not maintained personal hygiene than Non-Infectious group (28.7\%). Though this findings was not significant. Overcrowding was reported by $79.1 \%$ of the patients. Significantly (p-.01) proportion of Infectious diseases was more among those who lived in overcrowded condition (Infectious- $86.4 \%$, Non-Infectious-73.8\%).

\section{Discussion}

Type of skin diseases vary from country to country. In the same country it even varies from region to region. Skin diseases depend on different ecological, socio-economic, religious and environmental factors. This study presents the report of 225 patients from dermatology O.P.D of a tertiary care hospital in Kolkata. Proportion of child were one-fifth (19.5\%) of the studied patients. Most of the patients were from below < 44 years age group and females were more. Similar type of findings were also shown by Emmanouil K S et al,2006 in their study. ${ }^{10}$ But the study by G.S.Rao in a village in Kerala,2003,India found Male patients were more. ${ }^{(5)}$ In 
our study Muslim patients were more. The study by Florence Dalgard et al, 2007 showed that there were ethnic and gender differences in the reporting of skin complaints. ${ }^{11}$

Our study found among the total interviewed patients Noninfectious were more, similar to the study in Kerala (Non-Infectious 57.07\%, Infectious 43.4\%). ${ }^{5}$ Our study showed that among the Infectious group Scabies was the commonest form of dermatological presentation, followed by Tinea infection. Impetigo, Folliculitis, Boils, Pyoderma together constituted $8.9 \%$ of the studied patients. Among the Noninfectious group Dermatitis, Eczema, Miliaria Rubra, Lichen Simplex, Insect bite, Urticaria, Psoriasis were found. In the study done by Emmanouil K S \& others in Mediteranean island found Allergic dermatitis and Urticaria (35.7\%) were most commonly found cutaneous disorder followed by infectious diseases $(26.1 \%) .{ }^{10}$ Proportion of Insect bite $(10.2 \%)$ in this study was more compared to our study, reason of which may be due to different geographical area and our study was in a tertiary care hospital and this type of problem being an emergency situation might have been managed locally. The study in Dermatology O.P.D of Gauhati Medical college in India by Das KK,2003 found Eczema (23.1\%), Pyoderma (14.29\%), Fungal infections (14.24\%) and Psoriases (7.7\%) were the major skin diseases in that part of country. Study done by Th. Bijayanti Devi in North Eastern India also found Eczema (17.48\%),Fungal (17.19\%), Pyoderma (9.1\%) and Scabies (8.97\%) were the major pattern of skin morbidities. ${ }^{8}$ Fungal diseases $(20.6 \%)$ were the commonly found infection among children reported by Nuzhat Yasmeen,2005 in their study in Pakistan. ${ }^{(13)}$ The study in the tertiary care hospital in Kerala by N. Asokan, 2009 found Fungal infection (18.74\%), Bacterial (6.74\%), Parasitic (4.31\%) were commonly found among Infectious skin disorder and Eczema (21.83\%), Papulosquamous (12.3\%), Psoriasis (7.75\%) were the Noninfectious category. ${ }^{14}$ Another study in West Bengal by Sudip Das \& others in Dermatology O.P.D. of a Tertiary care hospital found proportion of different skin diseases more than the present study. ${ }^{15} \mathrm{We}$ found leprosy patients were $2.7 \%$, which was similar to the other study ${ }^{3}$, less than the other study ${ }^{15}$ and more than other studies. ${ }^{5,8,14}$ This study showed that the causes of infectious skin diseases for which the patients paid recurrent visits, some health education should also be given along with medical treatment to reduce the disease burden.

This study showed that type of skin diseases significantly varied with socio-economic classes. Reason is that patients coming from lower socio-economic category had low awareness, might live in overcrowded condition with poor environmental sanitation. As this study showed that infectious dermatoses were significantly (p-.00) more with overcrowded condition. Infectious skin diseases were more significantly among current tobacco user. Reason of which could not be explained, needs further research. This study showed that infectious cutaneous disorder was significantly more among those who had history of contact. It is quite likely if the pathogenesis of infectious diseases is thought of. Though the proportion of infectious diseases was more among those not maintained their personal hygiene nor had insufficient water but they were not statistically significant. Some of our study findings were also supported by WHO in their report that three main factors have been generally responsible for the high prevalence and incidence of common skin diseases in developing areas: a low level of hygiene, differential access to water, overcrowding and climatic factor. ${ }^{4}$

\section{Conclusion}

The findings of this study highlight the fact that along with medical treatment, health education is also necessary to curb the spread of cutaneous diseases, reduce the associated morbidity and improve the health status of the population.

VI. Tables

Table 1: Background characteristics of the interviewed patients $(\mathrm{n}=225)$

\begin{tabular}{|l|l|c|}
\hline Variables & Number & Percentages \\
\hline Age in years & & \\
$\leq 14$ & 44 & 19.5 \\
$15-24$ & 80 & 35.6 \\
$25-34$ & 50 & 22.2 \\
$35-44$ & 31 & 13.8 \\
$45-54$ & 10 & 04.5 \\
$55-64$ & 07 & 03.1 \\
$\geq 65$ & 03 & 01.3 \\
Gender & & \\
Male & 109 & 48.4 \\
Female & 116 & 51.5 \\
Religion & & \\
Hindu & 100 & 44.4 \\
Muslim & 124 & 55.2 \\
Christians & 01 & 0.4 \\
\hline
\end{tabular}




\begin{tabular}{|c|c|c|}
\hline Literacy status & & \\
\hline Illiterate & 45 & 20.0 \\
\hline Primary & 35 & 15.5 \\
\hline Secondary & 107 & 47.6 \\
\hline Higher Secondary & 29 & 12.9 \\
\hline Graduate \& above & 09 & 4.0 \\
\hline \multicolumn{3}{|l|}{ Occupation } \\
\hline Homemaker & 58 & 25.8 \\
\hline Service & 07 & 03.1 \\
\hline Skilled Worker & 14 & 06.2 \\
\hline Unskilled Worker & 33 & 14.7 \\
\hline Student & 53 & 23.5 \\
\hline Business & 15 & 06.7 \\
\hline Professional & 02 & 0.9 \\
\hline Others* & 43 & 19.1 \\
\hline \multicolumn{3}{|l|}{ Socio-Economic Class@ } \\
\hline I & 09 & 04.0 \\
\hline II & 28 & 12.4 \\
\hline III & 79 & 35.1 \\
\hline IV & 79 & 35.1 \\
\hline $\mathrm{V}$ & 30 & 13.4 \\
\hline
\end{tabular}

*others include children, adolescents, older persons. ${ }^{\circledR}$ B.G Prosad scale

Table 2: Interviewed patients according to the pattern of skin disorder (225)

\begin{tabular}{|l|l|l|}
\hline Pattern of skin disorder & Number & Percentage \\
\hline Infectious Dermatoses (n=103) & & \\
Scabies & 46 & 20.3 \\
Tinea & 24 & 10.7 \\
Impetigo & 08 & 03.6 \\
Folliculitis & 05 & 02.2 \\
Boils & 03 & 01.3 \\
Pyoderma & 04 & 01.8 \\
Leprosy & 06 & 02.7 \\
Candidiasis & 03 & 01.3 \\
Acute Paronychia & 02 & 00.9 \\
Chicken Pox(1),Pubic lice(1) & 02 & 00.9 \\
& & \\
Non-Infectious Dermatoses (n=122) & & \\
Acne & 28 & 12.4 \\
Dermatitis & 22 & 09.8 \\
Eczema & 16 & 07.1 \\
Miliaria Rubra & 09 & 04.0 \\
Lichen Simplex & 09 & 04.0 \\
Pompholix & 08 & 03.6 \\
Insect Bite & 07 & 03.1 \\
Urticaria & 06 & 02.7 \\
Psoriasis & 06 & 02.7 \\
Keratosis & 03 & 01.3 \\
Pruritus & 02 & 00.9 \\
Keloid (1), Leucoderma (1), Callosity(1), & \\
Naevus (1),Traumatic Injury(1),Alopecia(1) & & 02.7 \\
\hline
\end{tabular}


Table 3: Distribution of the study population according to the type of skin disorder and frequency of visit to this health facility $(n=225)$

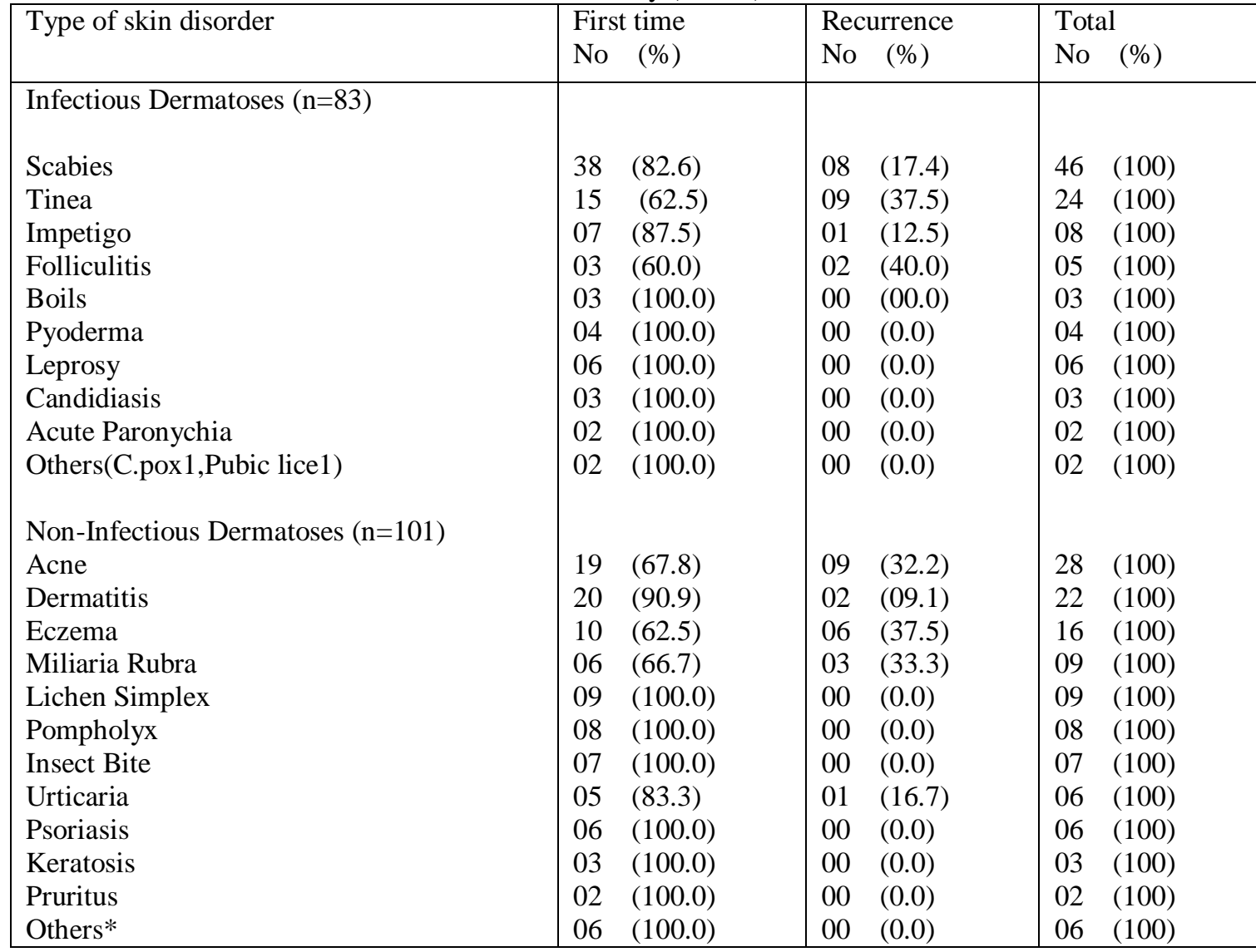

* Others include keloid, leucoderma, callosity, naevus, traumatic injury, alopecia each 1 in no.

Table 4: Distribution of the study subjects according to the type of disease and relation of some sociodemographic, environmental variables $(n=225)$

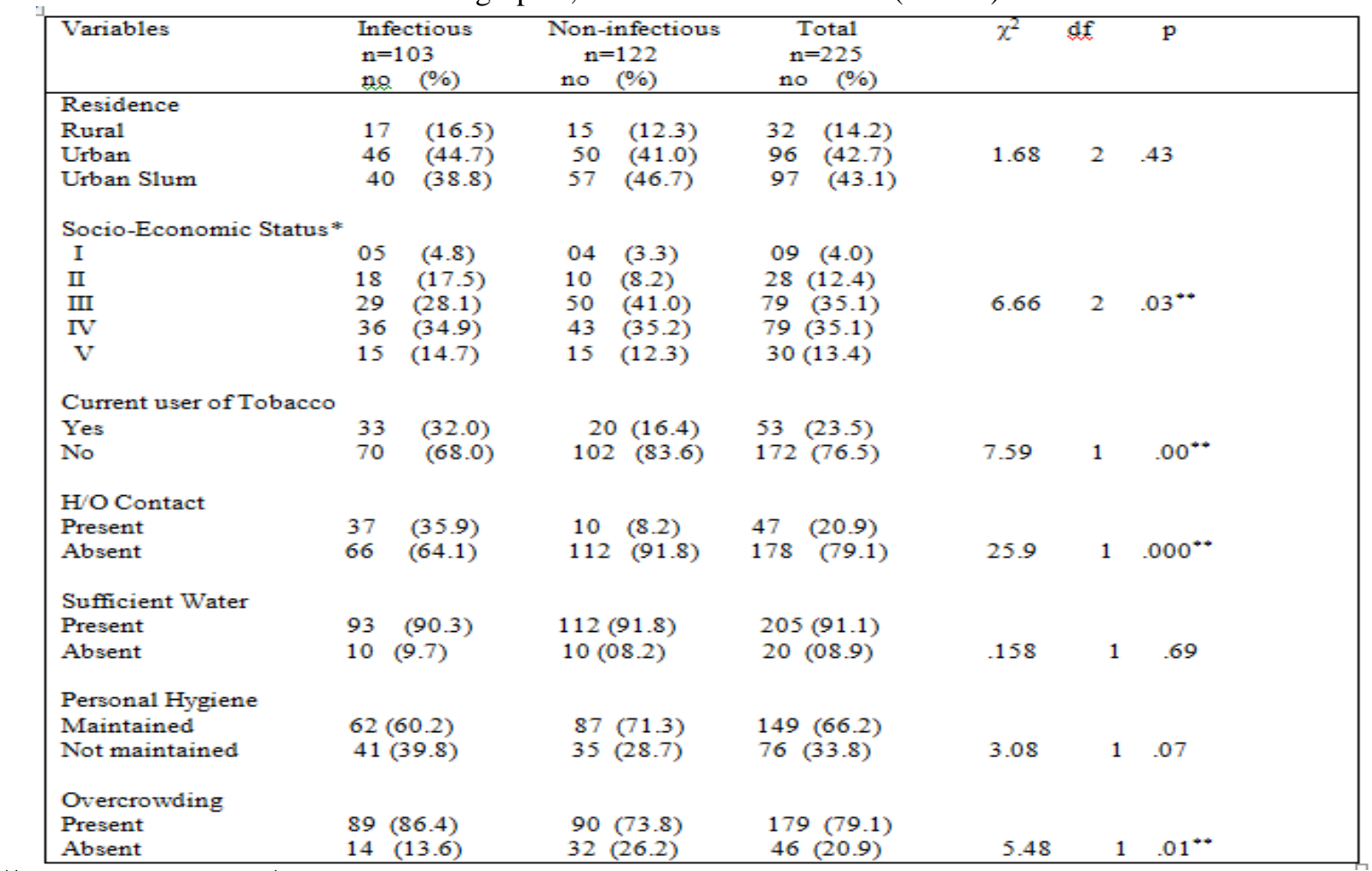

${ }^{* *}$-significant value, ${ }^{*}$ clubbing has been done between SE class I \&II and IV \&V. 


\section{References}

[1] O.M.V Schofield, J.A.A Hunter. Diseases of the skin in Davidson's Principles \& Practice of Medicine,21 ${ }^{\text {st }}$. Edition,.Edinburg,U.K.Publisher Elsevier. 2011:1376.

[2] Roderick Hay, Sandra E, Bendeck,Suephy Chen and others. Skin diseases in disease control priorities in developing countries. [accessed online www.ncbi.nlm.nih.govlbooks 1 KBK11733,24th.November 2011].p.708

[3] Das K K. Pattern of dermatological diseases in Gauhati Medical College \& Hospital, Guwahati. IJDVL 2003;69(1):16-18.

[4] Epidemiology and management of common skin diseases in children in developing countries. Department of Child and Adolescent Health \& Development. WHO 2005.1-62[WHO\FCH\CAH\05.12].

[5] G. S. Rao, S S Kumar, Sandhya. Pattern of skin diseases in an Indian village. Indian Journal of Medical Science 2003;57(3):108-10.

[6] Abbas Zamania, Hoseen Mahjum. Prevalence of skin diseases in hamedan, Iran in 2002. Indian Journal of Dermatology, WB, 2005;50(4):208-11.

[7] D.D.Atraide, M.R.Akpa and I.O.George. The Pattern of Skin disorders in a Nigerian Tertiary Hospital. Journal of Public Health \& Epidemiology April 2011,3(4):177-81.

[8] Th. Bijayanti Devi, G. Zamzachin. Pattern of skin diseases in Imphal. Indian J Dermatology 2006;51(2):149-50.[accessed online http://www.e-ijd.org,sunday,Nov,04,2012.IP:116.203.171.236].

[9] AK Agarwal.Social classification:The need to update in the present scenario. Indian J Community Med 2008;33(1):50-1

[10] Symvoulakis E K,Krasagakis K, Komninos I D, Kastrinakis I, Lyronis I, Philaliyhis A, Tosea A D.Primary care and pattern of Skin diseases in a Mediteranean island. B M C Family Practice 2006,7:6. [accessed online http: Ilwww.biomedcentral.com\1471229617\6].

[11] Dalgard F, Holm J, Svensson A, Kumar B, Sundby J. Self reported skin morbidity and ethnicity: a population based study in a western community. B M C Dermatology, June 2007,7:4.

[12] Dalgard F, Svensson A, Holm J, Sundby J. Self reported skin morbidity in Oslo: associations with socio-demographic factors among adults in a cross-sectional study.Br J Dermatol 2004,151:452-57.

[13] Nuzhat Yasmeen, Mohammad Riaz Khan. Spectrum of Common Childhood Skin diseases:a single centre experience. JPMA, Feb 2005.

[14] N Asokan, Priya Prathap, Ajithkumar K, Ambooken Betsy, Binesh V G, George S. Pattern of skin diseases among patients attending a tertiary care teaching hospital in Kerala. Indian J Dermatol Venerol Leprol Sept-Oct 2009;75(5):517.

[15] Sudip Das, Tapash Chatterjee. Pattern of skin diseases in a peripheral Hospital's Skin O.P.D:A Study of 2550 patients. Indian J Dermatol 2007;52(2):93-5.[accessed online http://www.cjid.org-nov04.2012 IP:116.203.171.236.]. 